\title{
De nouveaux gènes de facteurs de croissance de la famille CCN sont réglés par la voie de Wnt-1 dans le cancer du côlon
}

Les proto-oncogènes Wnt sont des protéines glycosylées capables, via leurs récepteurs membranaires, d'induire un signal intracellulaire impliqué dans divers processus au cours du développement tels que l'adhérence, la prolifération et la polarisation cellulaires [1] mais aussi le déterminisme des cellules. Le signal issu de Wnt est aujourd'hui relativement bien connu $[2,3]$ : fixation à son récepteur Frizzled puis recrutement membranaire de la protéine Dishevelled; l'activité constitutive de GSK3 $\beta$, glycogène synthase kinase, est alors inhibée ce qui provoque une stabilisation de la $\beta$-caténine. Cette dernière, dont la quantité semble réglée par le produit du gène suppresseur de tumeurs APC (adenomatous polyposis gene), peut alors interagir avec le facteur TCF/Lef1 pour activer la transcription. Jusqu'à présent, seules des mutations sur APC ou sur la $\beta$-caténine avaient pu être reliées à la tumorigenèse de certains carcinomes $[4,5]$. Aucune autre cible de la voie Wnt impliquée dans le cancer n'avait été identifiée jusqu'à présent. Tout récemment, ont été clonés [6] trois nouveaux gènes cibles de la voie de Wnt-1 par hybridation soustractive entre deux banques d'ADN issues de la même lignée cellulaire transformée ou non par Wnt-1. Les trois protéines nommées WISP-1, -2, -3 ont entre $30 \%$ et $40 \%$ d'identité globale entre elles et possèdent le même type d'homologie avec divers membres de la famille des facteurs de croissance CCN (cystein-rich connective), à savoir: (1) le CTGF (facteur de croissance du tissu conjonctif) qui est mitogène pour les fibroblastes et induit par le TGF $\beta$ [7]; (2) cyr61, une molécule de la matrice extracellulaire qui intervient dans l'adhérence, la migration et la prolifération cellulaires, mais aussi dans l'angiogenèse et la croissance des tumeurs [8] ; (3) nov (pour neuro- blastoma overexpressed), une protéine codée par un gène dit immédiat-précoce qui est associé à l'état de quiescence de la cellule et altéré dans la tumeur de Wilms [9].

Structuralement, les protéines WISP possèdent le modèle architectural de la famille CCN avec ses quatre domaines riches en cystéine: ainsi, celui du module amino-terminal des WISP est présent dans les IGF-BP (protéines de liaison des IGF, facteurs de croissance proches de l'insuline), alors qu'il a été montré par ailleurs que le CTGF fixait spécifiquement les IGF [10]. A l'opposé, dans le domaine carboxy-terminal, le groupe de dix cystéines qui semblent impliquées dans les phénomènes de dimérisation et de fixation à un récepteur, est présent dans tous les membres de la famille CCN, y compris WISP-1 et -3, mais pas WISP-2 qui en est dépourvu.

Un faisceau d'arguments suggère que les WISP sont des protéines sécrétées: présence dans les milieux de culture, absence de domaine transmembranaire, existence supposée d'une séquence-signal. En fait, alors que la famille des CCN et la protéine WISP1 ont des séquences différentes, elles partagent une fonctionnalité voisine: ce sont toutes des glycoprotéines sécrétées, riches en cystéine, présentant des domaines de fixation à l'héparine et capables de s'associer à la surface de la cellule ainsi qu'à la matrice extracellulaire.

L'expression tissulaire des trois gènes WISP varie d'une forme à l'autre suivant le tissu considéré mais sans spécificité stricte. Alors qu'ils sont localisés sur des chromosomes différents, WISP-1 a une localisation chromosomique très proche de celle de $\mathrm{c}-M Y C$, proto-oncogène souvent retrouvé amplifié dans les cellules cancéreuses en général et récemment ciblé dans la voie de Wnt au cours de la progression des cancers colo-rectaux [11]. De même, l'expression du gène WISP-
1 est amplifiée d'un facteur 2 dans $60 \%$ d'une série d'adénocarcinomes du côlon mais avec un profil différent de celui de c-MYC, de sorte que WISP1 ne peut faire partie de la même unité d'amplification génique (amplicon). Le gène WISP-2 est, quant à lui, amplifié dans plus de $90 \%$ de ces tumeurs (avec 2 à 4 copies), tandis que sur la même série de tumeurs, WISP-3 ne l'est jamais.

Enfin, par réaction de polymérisation en chaîne (PCR) quantitative sur les ARN présents dans les tissus tumoraux comparés aux normaux, les mêmes auteurs ont trouvé que les transcrits de WISP-1 et WISP-3 sont surexprimés dans respectivement $85 \%$ et $65 \%$ des tumeurs du côlon analysées, avec des taux variant de 2 à 25 fois pour WISP-1 et de 4 à 40 fois pour WISP-3. A l'opposé, toutes ces tumeurs, sauf une, ont des taux de transcrits de WISP-2 inférieurs au tissu normal, $50 \%$ des tumeurs présentant une baisse de 10 à 1000 fois. Une telle baisse de l'expression de WISP-2 dans les tumeurs du côlon est peutêtre à rapprocher de l'absence, dans la séquence de la protéine WISP-2, du motif carboxy-terminal de dimérisation/fixation au récepteur: en effet, WISP-2 pourrait jouer le rôle de suppresseur de tumeur, voire de variant endogène à comportement dominant-négatif sur les WISP-1 et -3, mais cela reste encore à prouver.

G.L'.A.

1. Wassef M. FGF-8, un signal impliqué dans l'organisation du tube neural. Med Sci 1996; 12: 991-5.

2. Romagnolo B. APC: de nouveaux partenaires, de nouveaux indices... Med Sci 1996; 12: 1109-10. 3. Deutsch J, Busson D. Des récepteurs pour les protéines de signalisation de la famille Wnt, enfin ? Med Sci 1997; 13: 222-4.

4. Cadigan KM, Nusse R. Wnt signaling: a common theme in animal development. Genes Dev 1997; 11 : 3286-305.

5. de la Coste A, Romagnolo B, Perret C. Une dérégulation de la voie de signalisation relayée par la $\beta$-caténine est impliquée dans l'hépatocarcinogenèse. Med Sci 1998; 14: 994-6.

6. Pennica D, Swanson TA, Welsh JW, et al. WISP genes are members of the connective tissue growth factor family that are up-regulated in Wnt- 
Références (suite)

1-transformed cells and aberrantly expressed in human colon tumors. Proc Natl Acad Sci USA 1998; 95 : 14717-22.

7. Grotendorst GR. Connective tissue growth factor: a mediator of TGF-beta action on fibroblasts. Cytokine Growth Factor Rev 1997; 8: 171-9.

8. Babic AM, Kireeva ML, Kolesnikova TV, Lau LF. CYR61, a product of a growth factor-inducible immediate early gene, promotes angiogenesis and tumor growth. Proc Natl Acad Sci USA 1998; 95: 6355-60.

9. Martinerie C, Huff V, Joubert I et al. Structural analysis of the human nov proto-oncogene and expression in Wilms tumor. Oncogene 1994; 9 : 2729-32.

10. Kim HS, Nagalla SR, Oh Y, et al. Identification of a family of low-affinity insulin-like growth factor binding proteins (IGFBPs) : characterization of connective tissue growth factor as a member of the IGFBP superfamily. Proc Natl Acad Sci USA 1997; 94 : 12981-6.

11. He TC, Sparks AB, Rago C, et al. Identification of $c-M Y C$ as a target of the APC pathway. Science $1998 ; 281$ : 1509-12.

\section{DRÈVES}

La protéase à sérine NES1, un nouveau suppresseur de tumeur du sein et de la prostate. On vient d'assigner le rôle de suppresseur de tumeur dans le tissu mammaire $(\mathrm{m} / \mathrm{s}$ 1997, $n^{\circ} 12$, p. 1471), à la protéase à sérine NES1 (normal epithelial cell specific-1) [1, 2]. Son gène a été mis en évidence par hybridation soustractive d'une lignée de cellules épithéliales mammaires humaines, $76 \mathrm{~N}$, et de la même lignée tumorale ayant subi une transformation cancéreuse induite par irradiation $\gamma$, 76R-30. L'analyse de la séquence d'un clone d'ADNc complet prédit une chaîne polypeptidique de $30 \mathrm{kDa}$. Les recherches dans les banques de données ont montré une similitude de $50-63 \%$ et une identité de 34-42\% avec plusieurs types de protéases à sérine: la famille de la trypsine, la famille des kallikréines (incluant l'antigène spécifique de la prostate (PSA), et la famille des protéases qui clivent les domaines kringle des facteurs de croissance. Tous les résidus d'acides aminés déterminants pour la liaison du substrat, la spécificité et l'activité catalytique des protéases à sérine sont conservés dans la séquence de la protéine NES1, suggérant une activité protéasique. Il s'agit en outre d'une protéine sécrétée. Son transcrit a été mis en évidence dans le thymus, la prostate, les testicules, l'intestin grêle, le côlon, le pancréas, le poumon, le cœur, avec le taux le plus élevé dans l'ovaire. Cette expression est réduite, voire complètement supprimée, dans les lignées cancéreuses mammaires. Par hybridation in situ, le gène NES1 a été localisé sur le chromosome 19, en $19 q 13.3$, région contenant d'autres gènes de protéases apparentées à NES1 (les kallikréines et le PSA) et remaniée dans les cancers humains [2]. L'expression stable de NES1 dans des lignées cellulaires de cancers du sein dépourvues de NES1 (lignée MDA-MB-231) en supprime l'oncogénicité, comme le montre l'absence d'apparition de tumeurs chez les souris nude auxquelles elles sont injectées. En raison de la localisation identique du gène codant pour le PSA sur le chromosome 19, des expériences similaires ont été réalisées avec des lignées cancéreuses de tissu prostatique et ont abouti à des résultats identiques: l'expression du transcrit de NES1 est réglée négativement au cours de la croissance tumorale dans les cancers de la prostate. Tous ces arguments confirment le rôle de NES1 en tant que gène suppresseur de tumeur. Alors que les protéases sont habituellement impliquées dans le processus invasif tumoral, NES1 a, au contraire, un effet inhibiteur sur la croissance tumorale [3]. Le mécanisme d'action, encore inconnu, pourrait être dû à un effet indirect de la protéase: inhibition d'un facteur de croissance ou de différenciation, ou bien activation d'autres inhibiteurs.

[1. Liu XL, et al. Cancer Res 1996; 56 : 3371-9.]

[2. Goyal J, et al. Cancer Res 1998; 58 : 4782-6.]

[3. Edwards DR, et al. Nature 1998; 394: 527-8.]

Un médicament anticancéreux actif sur les xénogreffes tumorales à MDR. L'un des problèmes majeurs posé aux cancérologues est la résistance, développée par les cellules tumorales, aux médicaments anticancéreux tels que le paclitaxel ou Taxol $^{\circledR}$, utilisé pour le traitement d'un large spectre de tumeurs solides. Cette résistance acquise est relayée par divers mécanismes dont la surexpression de la glycoprotéine $\mathrm{P}$ responsable du transport de ces médicaments [1]. L'ensemble de ces processus permet de faire acquérir à la cellule cancéreuse le phénotype de MDR (multi-drug resistance). De nouveaux anticancéreux, les macrolides naturels épothilones, tout en étant très éloignés en structure du paclitaxel, possèdent le même mécanisme d'action que celui-ci, à savoir la stabilisation des microtubules qui conduit à l'arrêt de la division cellulaire puis à la mort par apoptose [2]. Récemment, le laboratoire de S. Danishefsky (New York, USA) a montré que la famille des épothilones inhibe la croissance de toute une série de lignées tumorales à phénotype MDR pour le paclitaxel mais aussi pour la vinblastine, l'étoposide ou l'adriamycine [3]. A présent, le même laboratoire vient de montrer que, chez la souris athymique, la désoxy-épothilone $\mathrm{B}$ est capable de bloquer, parfois jusqu'à rémission totale, la croissance de tumeurs à MDR devenues résistantes au traitement par le paclitaxel, l'étoposide, la vinblastine ou l'adriamycine (doxorubicine) [4]. Ces résultats indiquent que les molécules anticancéreuses classiques n'induisent pas de résistance croisée avec l'épothilone. Cet énorme avantage se trouve renforcé par une synthèse chimique plus aisée que celle du paclitaxel et, surtout, par une meilleure solubilité aqueuse autorisant une meilleure biodisponibilité et donc une administration moins lourde au patient. Au total, toutes ces caractéristiques autorisent de réels espoirs en thérapeutique anticancéreuse.

[1. Lepage P, Gros P. Med Sci 1995; $11: 357-66$.

[2. Sackett D, Fojo T. Cancer Chemother Biol Resp Modif 1997 ; 17 : 59-79.]

[3. Chou TC, et al. Proc Natl Acad Sci USA 1998; 95: 9642-7.]

[4. Chou TC, et al. Proc Natl Acad Sci USA 1998; 95 : 15798-802.] 